\title{
Value of routine, non-targeted biopsies in the diagnosis of gastric neoplasia
}

\author{
B Cadman, M F Dixon, J I Wyatt
}

\begin{abstract}
Aim-To explore how often a diagnosis of gastric neoplasia is made on routine, nontargeted biopsies taken for determination of Helicobacter pylori status, compared with directed biopsies from endoscopically abnormal mucosa.

Methods-Records of all patients with a biopsy diagnosis of gastric cancer or dysplasia during a two year period were reviewed to determine whether the biopsy had been targeted at an area of mucosal abnormality, and whether there was any evidence of dysplasia or malignancy before endoscopy.

Results-Of the 8907 endoscopic examinations that included biopsy, histology showed malignancy in 115 cases and dysplasia in 20 . Of these, in 128 cases the biopsies were targeted from focal abnormal areas of mucosa, and six were from areas of diffuse mucosal thickening. In one case, adenocarcinoma was diagnosed in a patient with a "normal" endoscopic appearance; this patient was undergoing repeat endoscopy for previous dysplasia. Conclusions-Gastric malignancy or dysplasia was detected histologically in $1.5 \%$ of endoscopies that included biopsy. The performance of routine biopsies not targeted at a visible lesion from patients without previous diagnosis of neoplasia did not increase the detection of gastric malignancy. Such biopsies are indicated, however, if histological aspects of a patient's gastritis (such as atrophy or intestinal metaplasia) influence the clinical management, as in the treatment of helicobacter gastritis.

(f Clin Pathol 1997;50:832-834)
\end{abstract}

Keywords: gastric biopsy; gastritis; gastric cancer; Helicobacer pylori

Centre for Digestive

Diseases

M F Dixon

Department of Histopathology, St James's University Hospital, Leeds, UK J I Wyatt

Correspondence to: Dr Wyatt, Consultant Histopathologist, St James's University Hospital NHS Trust, Beckett Street, Leeds LS9 7TF, UK.

Accepted for publication 1 July 1997

During the past decade, the clinical significance of helicobacter gastritis has become recognised widely. At endoscopy, gastric biopsies are sent for histology for three possible reasons: to investigate localised mucosal lesions such as ulcers and tumours, to determine whether there is any gastritis present, and to establish the patient's helicobacter status. Both the number of upper gastrointestinal endoscopies performed annually, and the proportion of examinations that include biopsy have increased greatly during the past 10 years, although the latter varies considerably among different institutions.
There is a wide range of diagnostic tests for Helicobacter pylori, both invasive (using material obtained at endoscopy) and non-invasive. ${ }^{12}$ Histology offers one of the most accurate tests for helicobacter status, and compared with the biopsy urease test (for example, the CLO test) and microbiological culture it has the advantage of being an endoscopy based test already routinely available in the hospital. In addition, histology yields information about the presence and type of gastritis. Consequently, endoscopic biopsies not directed at focal abnormalities of the mucosa are now used frequently to determine helicobacter status, and this has resulted in a large increase in biopsy workload for many histology departments.

In a local audit designed to investigate the change in requesting patterns for gastric histology, gastroenterologists cited the need to determine helicobacter status as the main reason for the increasing proportion of upper gastrointestinal endoscopies that included biopsies; the importance of not missing gastric neoplasia was a second reason. ${ }^{3}$ Replacement of histology for routine detection of $H$ pylor $i$ by the CLO test was acceptable clinically, but we were concerned that any reduction in requests for gastric biopsy histology might result in missed opportunities for early detection of gastric neoplasia.

The aim of the present study was to determine, in a centre with a very high biopsy rate at endoscopy, the risk of missing gastric neoplasia if the CLO test were to replace histology for routine detection of $H$ pylori.

\section{Methods}

A high proportion (> 90\%) of upper gastrointestinal endoscopies performed at the Leeds General Infirmary include mucosal biopsy of antrum and corpus, as recommended by the Sydney System, for characterisation of gastritis. ${ }^{4}$

Pathology records from this institution for

the period January 1994 to December 1995 were reviewed for all gastric biopsies coded as dysplasia, carcinoma, lymphoma, or other forms of neoplasia or malignancy. The endoscopy reports from these cases were reviewed to determine whether the biopsies showing neoplasia had been targeted from an endoscopically visible lesion or had been taken as a "routine" biopsy for helicobacter status. The patients' notes were reviewed to determine whether there was a high index of suspicion for gastric neoplasia before endoscopy, based on previous histological or radiological findings. 
Table 1 Relation of histological diagnosis to endoscopic appearance and presence of targeted lesion

\begin{tabular}{llll}
\hline Histology and diagnosis & $\begin{array}{l}\text { Targeted biopsy } \\
\text { of lesion }\end{array}$ & Diffuse abnormality & $\begin{array}{l}\text { No visible } \\
\text { abnormality }\end{array}$ \\
\hline $\begin{array}{l}\text { Adenocarcinoma }(\mathrm{n}=109) \\
\quad \begin{array}{l}\text { Malignancy suspected at time } \\
\text { of endoscopy ( }=85)\end{array}\end{array}$ & 102 & 5 & 2 \\
$\begin{array}{l}\text { Malignancy not suspected at } \\
\text { time of endoscopy (n=24) }\end{array}$ & 78 & 5 & $2{ }^{\star} \dagger$ \\
High grade dysplasia (n=8) & 24 & 0 & 0 \\
Lymphoma (n=4) & 8 & 0 & 0 \\
Carcinoid tumour $(\mathrm{n}=2)$ & 4 & 0 & 0 \\
\hline
\end{tabular}

*Thickened folds reported on barium meal; tmultiple biopsies because of previous diagnosis of dysplasia.

In addition, a representative sample of 200 consecutive endoscopy reports was reviewed to determine the proportion of examinations at which a mucosal abnormality was recorded.

\section{Results}

During the two year study period, gastric biopsies from 8907 endoscopic examinations were sent to this department. Of these, $115(1.3 \%)$ showed malignancy, and $20(0.2 \%)$ showed dysplasia, which was high grade in eight and low grade in 12 . Of the malignant cases, 109 (94.8\%) were adenocarcinomas, four $(3.5 \%)$ were lymphomas, and two $(1.7 \%)$ were carcinoid tumours.

The summary of the patients' history and endoscopic findings is shown in table 1 . One hundred and two of 109 adenocarcinomas were diagnosed from biopsies targeted on a distinct endoscopic lesion, as were all 20 of the dysplasias, and all of the carcinoid tumours and lymphomas. A further six biopsies were taken from diffusely abnormal gastric mucosa suggestive of diffuse infiltrating adenocarcinoma (linitis plastica) as seen endoscopically $(n=5)$ or on ultrasound scan $(n=1)$. One patient had early gastric cancer diagnosed in a random biopsy with "normal" endoscopic appearances; however, he had been referred following a previous biopsy showing dysplasia in another hospital. Only one of 18 random biopsies from this patient showed adenocarcinoma, and none showed dysplasia.

A review of the patients' case notes showed that in 106 patients the histological result was the first diagnosis of malignancy while 29 examinations were performed because of previous evidence of neoplasia. For 11 of these 29 patients, the endoscopy was performed as a follow up for previous gastric malignancy, while in four malignancy was suspected on the basis of radiology. Fourteen endoscopies were performed because of previous histological findings: three had had a biopsy suspicious of adenocarcinoma and 11 had previously shown dysplasia (nine high grade, two low grade). Of these 11, adenocarcinoma was diagnosed on repeat biopsy in five (including two with low grade dysplasia); three again showed high grade dysplasia and three showed low grade dysplasia. In none of the 135 cases of malignancy or dysplasia was the primary diagnosis made on the basis of an endoscopic biopsy taken from unremarkable mucosa, or of mucosa simply showing "gastritis" endoscopically.
A review of the sample of 200 consecutive endoscopy reports showed that a mucosal abnormality was recorded in $53(26.5 \%)$. Of these, histology showed a focal lesion such as polyp, ulcer, or carcinoma in 26; while 27 revealed gastritis of various patterns. In 10 of these endoscopy reports, gastric carcinoma was seen $(n=5)$ or suspected $(n=5)$. Histology confirmed the five cases diagnosed by their endoscopic appearance, and diagnosed adenocarcinoma in one of the five suspicious cases, together with one case where an endoscopically "benign" ulcer was biopsied. Follow up biopsies obtained from the other patients with appearances suspicious of malignancy have not shown malignancy. Thus, this subgroup showed malignancy in seven of $53(13.2 \%)$ patients with a mucosal abnormality recorded at endoscopy.

\section{Discussion}

The use of upper gastrointestinal endoscopy in the investigation of patients with dyspepsia has increased in recent years. Although strategies for reducing this by screening patients for helicobacter have been proposed, ${ }^{5}$ they have not been adopted widely because of the value that gastroenterologists place on visualisation of the mucosa, especially for the early detection of neoplasia. Gastric biopsies are taken during endoscopy to confirm or exclude malignancy or premalignant change, to diagnose gastritis, and determine helicobacter status.

The recent increase in the detection of early gastric cancer in the UK in some centres has been achieved by improvement in endoscopic technique, and a high index of suspicion resulting in biopsy of any focal gastric mucosal lesion. ${ }^{6}$ Alternative tests are available for establishing helicobacter status, whereas there is no alternative to histology for the diagnosis of gastric neoplasia.

Histology is among the most accurate methods for determining helicobacter status, but performs best when multiple biopsies are taken and an additional stain, such as Giemsa or Warthin-Starry is used to highlight the organisms. ${ }^{4}$ When bacterial load is small, the hunt for helicobacter is laborious. Good results with histology have been obtained in the context of clinical trials, where histology is likely to be optimum. Where helicobacter numbers are small, or where only haematoxylin and eosin is used, with no additional stain, histology suffers from considerable interobserver variation, resulting in both false negatives and false positives. ${ }^{78}$ In these circumstances, the biopsy urease test (for example, the CLO test) may well be more accurate, and is certainly quicker and cheaper than histology. ${ }^{2}$

Helicobacter gastritis differs among individuals in severity and topography, and the pattern of gastritis bears some relation to an individual patient's risk of ulcer disease and cancer. ${ }^{49}$ Therefore, to obtain maximum information about gastritis, two biopsies each from the antrum and corpus are recommended. These biopsies allow a detailed classification of inflammatory disorders of the gastric mucosa, both those related to and independent of 
helicobacter infection. The latter would include reactive gastritis and lymphocytic gastritis, the clinical significance of which is still becoming apparent. Individual gastroenterologists will vary in the importance they place on obtaining gastric histology. Clinical indications for treating $H$ pylori infection are still evolving $^{10}$; and for clinicians who would treat any patient in whom the infection is detected, histological characterisation of gastritis is unnecessary. However, if the particular histological characteristics of the gastritis, such as severity or presence of intestinal metaplasia, affect the decision to treat the patient, ${ }^{11}$ then this could imply that the appropriate biopsies for histopathology are taken at each endoscopy. In this context, the updated version of the Sydney System recommends that an additional biopsy is taken from the incisura as the site of predilection for intestinal metaplasia and epithelial dysplasia ${ }^{12}$; routine biopsies from this site were not included in our study. The incorporation of multiple protocol biopsies as routine in all endoscopies would have large resource implications for gastroenterology and histopathology departments.

In summary, we reviewed 135 cases of malignancy or dysplasia diagnosed on gastric biopsy over a two year period during which 8907 endoscopies with biopsy were performed. All cases were detected from biopsies of endoscopically abnormal mucosa, apart from one where gastric mapping was being performed for previously diagnosed dysplasia. No case was an unsuspected diagnosis made from a "routine" biopsy for detection of helicobacter gastritis. We conclude that the use of techniques other than histopathology to determine $H$ pylori status would not have reduced the number of malignancies detected during the study period.

1 Barthel JS, Everett ED. Diagnosis of Campylobacter pylor infections: The "gold standard" and the alternatives. Rev Infect Dis 1990;12(suppl 1):S107-14.

2 Cutler AF, Havstad S, Ma CK, Blaser MJ, Perez-Perez GI, Schubert TT. Accuracy of invasive and noninvasive tests to diagnose Helicobacter pylori infection. Gastroenterology 1995;109:136-41.

3 Davies GR, Wyatt JI. Reducing the gastric biopsy histopathology workload through audit [abstract]. Gut 1995; 37(suppl 2):A8.

4 Price AB. The Sydney System: histological division. 7 Gastroenterol Hepatol 1991;6:209-22.

5 Sobala AB, Crabtree JE, Pentith JA, Rathbone BJ, Shallcross TM, Wyatt JI. Screening dyspepsia by serology to Helicobacter pylori. Lancet 1991;338:94-6.

6 Sue-Ling HM, Johnston D, Martin IG, Dixon MF, Lansdown MRJ, McMahon MJ, et al. Gastric cancer: Lansdown MRJ, McMahon MJ, et al. Gastric
curable disease in Britain. BMf 1993;307:591-6.

7 Christensin AH, Gjorup T, Hilden J, Feneger C, Henriksen $\mathrm{B}$, Vyberg $\mathrm{M}$, et al. Observer homogeneity in histologica hiagnosis of Helicobacter pylori. Scand $\mathcal{f}$ Gastroenterol 1992;27:933-9.

8 Kolts BE, Joseph B, Achem SR, Bianchin T, Monteiro C. Helicobacter pylori detection: a quality and cost analysis. Am f Gastroenterol 1993;88:650-5.

9 Wyatt JI. Histopathology of gastroduodenal inflammation: the impact of Helicobacter pylori. Histopathology 1995;26: the im.

10 Guidelines in gastroenterology: dysplasia management guidelines. London: British Society of Gastroenterology, 1996.

11 Malfertheiner P, Megraud F, O'Morain C, Berll D, Bianch Porro G, Deltenre M, et al. Current European concepts in the management of Helicobacter pylori infection-the the management of Helicobacter pylori infection-the 1997;9:1-2.

12 Dixon MF, Genta RM, Yardley JH, Correa P, and the participants. Classification and grading of gastritis. The updated Sydney System. Am $\mathcal{F}$ Surg Pathol 1996;20:116181 . 